\title{
FIABILIDAD Y REPRODUCTIVIDAD DE LOS CUESTIONARIOS DE ACTIVIDAD FÍSICA PAQ-C Y PAQ-A EN ESTUDIANTES DE ENSEÑANZA BÁSICA Y MEDIA DE LA CIUDAD DE TALCA
}

\section{RELIABILITY AND REPRODUCTIVITY OF THE PAQ-C AND PAQ-A PHYSICAL ACTIVITY QUESTIONNAIRES IN STUDENTS IN BASIC AND SECONDARY EDUCATION OF THE CITY OF TALCA}

\author{
CÉSAR FAÚNDEZ CASANOVA* \\ JAIME VÁSQUEZ** \\ RICARDO SOUZA*** \\ MARCELO CASTILLO**** \\ FRANKLIN CASTILLO***** \\ JOSÉ PÉREZ****** \\ JAVIERA GUZMÁN*******
}

Rec.: 02-01-2020. Acept.: 10-11-2020. Publ.: 18-12-2020

DOI: http://doi.org/10.29035/ucmaule.59.56

\section{RESUMEN}

Los cuestionarios PAQ-C y PAQ-A tienen como objetivo medir los niveles de actividad física, últimos 7 días, que presentan los estudiantes. A través de esta investigación se pretende determinar la fiabilidad y reproductividad del cuestionario en su versión en español. Se estudió a 192 sujetos de los cuales 95 eran hombres correspondientes al y 97 eran mujeres. Se consideró en el estudio a niños y adolescentes cuyas edades iban desde los 9 años hasta los 18 años. Para valorar la Actividad física (AF) se utilizó los cuestionarios PAQ-A (Physical Activity Questionnaire for Adolescents) y PAQC (Physical Activity Questionnaire for Children) los cuestionarios se aplicaron a los sujetos de forma auto administrada en el que los adolescentes dispusieron de 30 minutos para responder. El instrumento fue aplicado en dos instancias, teniendo un intervalo de 7 días entre las dos mediciones (test y re-test). De acuerdo a la investigación, es importante señalar que los cuestionarios PAQ-C y PAQ-A tienen una consistencia interna más que aceptable, transformándose en un cuestionario que contribuye de forma segura y confiada al evaluar los niveles de actividad física de niños y adolescentes durante el periodo escolar anual.

Palabras clave: Actividad física, salud, PAQ-C, PAQ-A, Niveles de Actividad Física.

* Universidad Católica del Maule, Talca, Chile. https://orcid.org/0000-0003-4501-4169. cfaundez@ucm.cl

** Universidad Católica del Maule, Talca, Chile. https://orcid.org/0000-0003-0597-793X jvasquez@ucm.cl

*** Universidad Católica del Maule, Talca, Chile. https://orcid.org/0000-0003-1715-9213 rsouza@ucm.cl

**** Universidad Católica del Maule, Talca, Chile. https://orcid.org/0000-0002-7482-1165 mcastillo@ucm.cl

***** Universidad Católica del Maule, Talca, Chile. https://orcid.org/0000-0001-9118-4340 fcastillo@ucm.cl

****** SportLife, Talca, Chile. joseignacioperez0612@gmail.com

******* FitnessPro Entrenamiento Funcional, Talca, Chile. fran18@hotmail.es 


\section{ABSTRACT}

The purpose of the PAQ-C and PAQ-A questionnaires is to measure the levels of physical activity. The last 7 days, presented by students. Through this research we try to determine the reliability and reproducibility of the questionnaire in its Spanish version. A total of 192 subjects were studied, of which 95 were men, and 97 were women. Children and adolescents whose ages ranged from 9 years to 18 years were considered in the study. To assess physical activity (PA), the questionnaires PAQ-A (Physical Activity Questionnaire for Adolescents) and PAQ-C (Physical Activity Questionnaire for Children) were used. The questionnaires were applied to the subjects in a selfadministered way in which the adolescents had 30 minutes to respond. The instrument was applied in two instances, having an interval of 7 days between the two measurements (test and re-test). According to the research, it is important to point out that the questionnaires PAQ-C and PAQ-A have a more than acceptable, transforming into a questionnaire that contributes in a safe and confident way when evaluating the physical activity levels of children and adolescents during the annual school period.

Key words: Physical activity, health, PAQ-C, PAQ-A, levels of physical activity.

\section{INTRODUCCIÓN}

"El ser humano desde sus orígenes está preparado para moverse, más aun, tiene la necesidad de hacerlo" (Escalante, 2011. p. 325). La práctica de actividad física hoy en día es cada vez menos frecuente en la población mundial, muy notoriamente en niños y adolescentes. Esto permite concluir que buena parte de esta población tenga riesgos de ser clasificados como sedentarios, lo que unido a malos hábitos alimenticios pueda provocar el aumento en diversas patologías (obesidad, enfermedades cardiovasculares, síndrome metabólico, etc.) que, en algunos casos se han convertido en un auténtico problema de salud a nivel país (Lobstein, Baur, \& Uauy, 2004).

La actividad física se define como "cualquier movimiento del cuerpo producido por el sistema musculoesquelético del que resulta un gasto energético" (Caspersen, Powell \& Chisterson, 1985), además de ser un agente relevante en la prevención de enfermedades crónicas no transmisibles como la diabetes, la obesidad, el síndrome metabólico, etcétera. (Faúndez, Vásquez, 
Faúndez, C. (2020). Fiabilidad y reproductividad de los Cuestionarios de Actividad Física PAQ-C Y PAQ-A en Estudiantes de Enseñanza Básica y Media de la Ciudad de Talca. UCMaule, 59, julio-diciem bre, 56-78.

DOI: http://doi.org/10.29035/ucmaule.59.56

Castillo, Souza \& Castillo, 2019). Es así, como en diferentes países se ha creado la necesidad de crear políticas de salud para generar instancias de promoción de actividad física entre la población (OMS, 2010).

Es fundamental practicar actividad física día a día, fomentar una rutina de movimiento y de actividades que ayuden a llevar una vida activa, relacionándola directamente con una alimentación saludable (Gobierno de Chile, 2017). Hoy en día existen programas de promoción de la actividad física y la salud, en donde se evidencia la importancia de la actividad física como promoción de salud, (Vidarte, Vélez, Sandoval \& Alfonso, 2011; Gobierno de Chile, 2017) sobre todo en edades tempranas -infancia y adolescencia-. Lo que se busca es promover la actividad física para lograr un desarrollo integral de niños y adolescentes y se ha utilizado con éxito para la prevención y tratamiento de la obesidad, la hipertensión y otras enfermedades crónicas (Sánchez et al, 2019). Las practicas saludables en relación a este tema, de forma guiada y segura, puede entregar a los participantes efectos beneficiosos en el desarrollo motor, cognitivo, afectivo, social, y prácticamente en todos los sistemas (Armstrong \& Welsman, 2007).

Es en edades tempranas en donde el generar buenas prácticas tiene influencias directas sobre la salud en la vida adulta (National Center for Chronic Disease Prevention and Health Promotion, Centres for Disease Control and Prevention, 1997; Perula de Torres, Lluch, Ruiz, Espejo, Tapi \& Mengual, 1998; Janz, Dawson \& Mahoney, 2000).

Algunos estudios realizados en gran parte de Europa y principalmente en España, en niños y adolescentes de 9 a 16 años, han detectado que los mayores niveles de actividad física se pueden presenciar en varones (Riddoch, Bo Andersen, Wedderkopp, Harro, Klasson-Heggebø, Sardinha, \& Ekelund, 2004; Martínez, Martínez, Pozo, Villagra, Calle, Marcos \& Veiga, 2009).

Para determinar los niveles de actividad física en la población existen variados instrumentos para precisar la ocupación de los sujetos en el contexto de una vida activa (Serón, Muñoz \& Lanas, 2010). Como en todo aspecto, algunos de estos instrumentos son más precisos que otros, pero a la vez involucran un mayor tiempo en su trabajo y análisis. Además, poseen un alto costo y se tornan inviables para valorar a grandes grupos de personas. 
Faúndez, C. (2020). Fiabilidad y reproductividad de los Cuestionarios de Actividad Física PAQ-C Y PAQ-A en Estudiantes de Enseñanza Básica y Media de la Ciudad de Talca. UCMaule, 59, julio-diciem bre, 56-78.

DOI: http://doi.org/10.29035/ucmaule.59.56

Existen otras alternativas que se suelen utilizar más en laboratorios o centros de salud. Aunque estas técnicas son más precisas, son más difíciles utilizar al evaluar a grandes poblaciones, como es el caso de calorimetría directa, técnica de doble agua marcada, los acelerómetros, podómetros y monitores de ritmo cardiaco. Ahora bien, se pueden encontrar los cuestionarios que son la herramienta más accesible y viable para valorar la actividad física que realizan niños y adolescentes cuando se desea la obtención de datos en grandes muestras (Welk, 2002).

Dentro de los cuestionarios más utilizados a nivel internacional se encuentran los formularios de la familia PAQ y para este grupo de edad (8 a 18 años), es el cuestionario de actividad para adolescentes PAQ-A (Physical Activity Questionnaire for Adolescents) y PAQ-C (Physical Activity Questionnaire for Children). (Martínez, Martínez, Pozo, Villagra, Calle, Marcos, \& Veiga, 2009). El PAQA y PAQ-C se incluyen dentro de la denominada "familia PAQ", que comprende cuestionarios muy similares para valorar la actividad física en tres grupos de edad: en niños de entre 8-12 años mediante el PAQ-C (Physical Activity Questionnaire for Children), en adolescentes entre 13-18 años con el PAQ-A, y en adultos usando el PAQ-AD (Physical Activity Questionnaire for Adults) (Martinez et al, 2009).

El objetivo de esta investigación es valorar la fiabilidad y reproductividad del PAQ-C y PAQ-A en su versión en español en estudiantes de enseñanza básica y media en escuelas municipalizadas de Talca

\section{METODOLOGÍA}

El estudio es descriptivo de corte transversal. Se estudió a 192 sujetos de los cuales 95 eran hombres -correspondientes al 49,5\% de la población total estudiada-, y 97 eran mujeres-correspondientes al 50,5\% del total de la población estudiada- de 2 colegios municipales de la región del Maule (Chile). La muestra fue calculada mediante las elecciones no probabilísticas por cuotas. Se consideró en el estudio a niños y adolescentes cuyas edades iban desde los 9 años hasta los 18 años.

Todos los niños y adolescentes hasta la fecha de la evaluación realizaban actividad física una vez por semana, fruto de las clases de educación física reglamentada por el Ministerio de Educación, que oscilaba entre 80-90 minutos por sesión. 
En todos los casos se obtuvo el consentimiento de los padres y/o de los apoderados de los niños y adolescentes, como el asentimiento por parte de los participantes, así como el permiso respectivo de la dirección de las instituciones educativas. Todo esto siguiendo los lineamientos éticos establecidos por el tratado de Helsinki.

\section{Procedimientos}

Para definir la edad cronológica se utilizó el registro de nacimiento de cada alumno (día, mes y año) y la fecha de la evaluación (día, mes y año) para calcular la edad decimal. Las fechas de nacimiento fueron verificadas a través de la ficha de matrícula de cada adolescente.

Para valorar la Actividad física (AF) se utilizaron los cuestionarios PAQ-A (Physical Activity Questionnaire for Adolescents) y PAQ-C (Physical Activity Questionnaire for Children). Estos fueron traducidos de los originales planteados por Kowalski, Crocker \& Donen (2004) y contrastado con la versión en español de Manchola-González, Bagur-Calafat, \& Girabent-Farrés (2017) y de Martínez et al (2009). Luego se les se les realizó una adaptación semántica siguiendo los lineamientos dados por Parasuraman, Zeithaml \& Berry (1988).

Los cuestionarios se aplicaron a los sujetos de forma autoadministrada. Los adolescentes dispusieron de 30 minutos para responder al inicio de la clase de educación física. El instrumento fue aplicado en dos instancias, teniendo un intervalo de 7 días entre las dos mediciones (test y retest). El retest se aplicó a todos los sujetos y en las mismas condiciones que el test, manteniendo al mismo encuestador.

\section{Análisis esta dístico}

La distribución normal de la muestra, en cada una de las variables de análisis, fue verificada a través de la prueba de normalidad Kolmogórov-Smirnov. Los datos recolectados se almacenarán en una base de datos en el programa Excel 2010, la cual será analizada mediante el programa estadístico SPSS versión 24. Para describir las variables, se empleará medidas de tendencia central (promedios) y de dispersión (desviación estándar [DS]), minino y máximo (MIN y MAX). Para verificar la fiabilidad y confiabilidad (consistencia interna) se aplicó el coeficiente de Alpha de Cronbach, considerando aceptable los rangos descritos por Campo \& 
Oviedo (2008). Para verificar las diferencias entre el test y retest se utilizó el coeficiente de correlación intraclase (ICC) y el intervalo de confianza de este al 95\% (IC 95\%) tomando como referencia los criterios descritos por Fless en 2004. En todos los casos se asumió la significación estadística con un p-valor < 0.05.

\section{RESULTADOS}

Los cuestionarios aplicados a los estudiantes desde 4 básico hasta 4 medio tuvieron una aceptación positiva por parte de los encuestados.

De una población total de 282 posibles encuestados, 192 completaron test y el retest, cumpliendo con los criterios de selectividad. 95 de los participantes eran varones -correspondientes al 49,5\% de la muestra total-, y 97 eran damas correspondientes al 50,5\% de la muestra total de estudiantes-. Como muestra la Tabla 1, se puede observar la selección de la muestra, la estadística en la edad y el número de participantes por nivel escolar.

La mayor cantidad de participantes se concentra en los niveles de 5 to año básico, en el cual la muestra total fue de 28 estudiantes, alcanzando un 14,6\% del total de la muestra. También se puede observar que en el nivel escolar de ler año medio la muestra total alcanzó el número de 26 estudiantes, equivalentes al 13,5\% del total de la muestra encuestada. 
Tabla 1

Selección de la muestra.

\begin{tabular}{|c|c|c|c|c|c|c|c|}
\hline \multirow[t]{2}{*}{ Curso } & & \multicolumn{2}{|c|}{ Masculino } & \multicolumn{2}{|c|}{ Femenino } & \multicolumn{2}{|c|}{ Total } \\
\hline & & Población & Muestra & Población & Muestra & Población & Muestra \\
\hline \multirow{2}{*}{$4^{\circ}$ Básico } & Recuento & 20 & 10 & 20 & 14 & 40 & 24 \\
\hline & $\%$ del total & $13,69 \%$ & $5,2 \%$ & $14,70 \%$ & $7,3 \%$ & $14,18 \%$ & $12,5 \%$ \\
\hline \multirow{2}{*}{$5^{\circ}$ Básico } & Recuento & 12 & 12 & 16 & 16 & 28 & 28 \\
\hline & $\%$ del total & $8,21 \%$ & $6,3 \%$ & $11,76 \%$ & $8,3 \%$ & $9,92 \%$ & $14,6 \%$ \\
\hline \multirow{2}{*}{$6^{\circ}$ Básico } & Recuento & 19 & 8 & 12 & 10 & 31 & 18 \\
\hline & $\%$ del total & $13 \%$ & $4,2 \%$ & $8,82 \%$ & $5,2 \%$ & $10,99 \%$ & $9,4 \%$ \\
\hline \multirow{2}{*}{$7^{\circ}$ Básico } & Recuento & 14 & 12 & 13 & 12 & 27 & 24 \\
\hline & $\%$ del total & $9,58 \%$ & $6,3 \%$ & $9,50 \%$ & $6,3 \%$ & $9,57 \%$ & $12,5 \%$ \\
\hline \multirow{2}{*}{$8^{\circ}$ Básico } & Recuento & 19 & 17 & 7 & 7 & 26 & 24 \\
\hline & $\%$ del total & $13 \%$ & $8,9 \%$ & $5,14 \%$ & $3,6 \%$ & $9,21 \%$ & $12,5 \%$ \\
\hline \multirow{2}{*}{$7^{\circ}$ Medio } & Recuento & 17 & 12 & 18 & 14 & 35 & 26 \\
\hline & $\%$ del total & $11,64 \%$ & $6,3 \%$ & $13,20 \%$ & $7,3 \%$ & $12,41 \%$ & $13,5 \%$ \\
\hline \multirow{2}{*}{$2^{\circ}$ Medio } & Recuento & 12 & 8 & 18 & 9 & 30 & 17 \\
\hline & $\%$ del total & $8,21 \%$ & $4,2 \%$ & $13,20 \%$ & $4,7 \%$ & $10,63 \%$ & $8,9 \%$ \\
\hline \multirow{2}{*}{$3^{\circ}$ Medio } & Recuento & 18 & 11 & 19 & 10 & 37 & 21 \\
\hline & $\%$ del total & $12,32 \%$ & $5,7 \%$ & $13,90 \%$ & $5,2 \%$ & $13,12 \%$ & $10,9 \%$ \\
\hline \multirow{2}{*}{$4^{\circ}$ Medio } & Recuento & 15 & 5 & 13 & 5 & 28 & 10 \\
\hline & $\%$ del total & $10,27 \%$ & $2,6 \%$ & $9.5 \%$ & $2,6 \%$ & $9,92 \%$ & $5,2 \%$ \\
\hline \multirow{2}{*}{ Total } & Recuento & 146 & 95 & 136 & 97 & 282 & 192 \\
\hline & $\%$ del total & $51,70 \%$ & $49,5 \%$ & $48,30 \%$ & $50,5 \%$ & $100 \%$ & $100,0 \%$ \\
\hline
\end{tabular}

En la Tabla 2 se logra observar las características de la muestra, en donde se encuentra que el número total de la muestra fue de 192 estudiantes, 95 de ellos son de sexo masculino y 97 de sexo femenino. También se puede encontrar que el promedio de edad en hombres es de 13,77 años y en mujeres es de 13,43 años. La edad mínima encontrada entre todos los participantes es de 9,0 años 
Faúndez, C. (2020). Fiabilidad y reproductividad de los Cuestionarios de Actividad Física PAQ-C Y PAQ-A en Estudiantes de Enseñanza Básica y Media de la Ciudad de Talca. UCMaule, 59, julio-diciem bre, 56-78.

DOI: http://doi.org/10.29035/ucmaule.59.56

correspondientes a una dama, y la edad mayor es de 18,8 años correspondiente a un varón.

Tabla 2

Caracterización de la muestra.

\begin{tabular}{cccccc}
\hline Edad & $\mathrm{N}$ & $\mathrm{X}$ & $\mathrm{DE}$ & Mínimo & Máximo \\
\hline Masculino & 95 & 13,77 & 2,49 & 9,4 & 18,8 \\
\hline Femenino & 97 & 13,43 & 2,61 & 9,0 & 17,7 \\
\hline Total & 192 & 13,60 & 2,55 & 9,0 & 18,8 \\
\hline
\end{tabular}

La Tabla 3 muestra los coeficientes de Cronbach (alfa) para la primera y segunda medición con el fin de fiabilidad de los cuestionarios PAQ-C y PAQ-A. Se observa que el cuestionario PAQ-C logro una consistencia interna de 0,844 en la primera medición y 0,843 en la segunda medición, mientras que el cuestionario PAQ-A logro una consistencia interna de 0,853 en la primera medición y 0,852 en la segunda medición. En ambos casos, se interpreta como un coeficiente de cronbach aceptable.

Tabla 3

Valores del coeficiente de Cronbach (alfa) para fiabilidad del cuestionario de actividad física.

\begin{tabular}{|c|c|c|c|c|}
\hline \multirow{2}{*}{ Ítems } & \multicolumn{2}{|c|}{$P A Q-C$} & \multicolumn{2}{|c|}{$P A Q-A$} \\
\hline & test & re-test & test & re-test \\
\hline 1. Actividad física en tiempo libre: Relación de actividades & 0,841 & 0,839 & 0,848 & 0,846 \\
\hline 2. Esfuerzo físico más intenso en las clases de educación física & 0,855 & 0,854 & 0,857 & 0,854 \\
\hline 3. Actividades realizadas en el marco recreo escolar & 0,832 & 0,831 & & \\
\hline 4. Ocio activo / deporte / ejercicio: físico en la mañana & 0,845 & 0,844 & 0,845 & 0,842 \\
\hline 5. Ocio activo / deporte / ejercicio: físico en la tarde & 0,811 & 0,810 & 0,846 & 0,844 \\
\hline 6. Ocio activo / deporte / ejercicio: físico en el período de la noche & 0,807 & 0,805 & 0,824 & 0,823 \\
\hline 7. Ocio activo / deporte / ejercicio: el fin de semana & 0,822 & 0,821 & 0,812 & 0,810 \\
\hline 8. Intensidad del esfuerzo físico semanal & 0,820 & 0,819 & 0,828 & 0,826 \\
\hline 9. Frecuencia diaria de actividad física más intensa & 0,813 & 0,814 & 0,820 & 0,820 \\
\hline Puntuación total PAQ & 0,844 & 0,843 & 0,853 & 0,852 \\
\hline
\end{tabular}


Faúndez, C. (2020). Fiabilidad y reproductividad de los Cuestionarios de Actividad Física PAQ-C Y PAQ-A en Estudiantes de Enseñanza Básica y Media de la Ciudad de Talca. UCMaule, 59, julio-diciem bre, 56-78.

DOI: http://doi.org/10.29035/ucmaule.59.56

En la Tabla 4 se observa la fiabilidad y reproductividad de los cuestionarios PAQ-C y PAQ-A, en donde se tuvieron en cuenta las dos mediciones test y re-test, por lo tanto se calculó el ICC en los cuales los valores de fiabilidad fueron superiores a 0.8 con intervalos de confianza entre 0.8 - 0.9, lo cual sigue indicando un ICC considerablemente bueno, tanto en hombres como en mujeres y el total de la muestra, lo que indica una buena reproductibilidad y una excelente fiabilidad de los cuestionarios.

Tabla 4 Fiabilidad y reproducibilidad del cuestionario de actividad física correlación intraclase.

\begin{tabular}{lcccc}
\hline \multirow{2}{*}{\multicolumn{1}{c}{ Ítems }} & \multicolumn{2}{c}{ PAQ-C } & \multicolumn{2}{c}{ PAQ-A } \\
\cline { 2 - 5 } & ICC & IC 95\% & ICC & IC 95\% \\
\hline Puntuación total PAQ Varones & 0.918 & $0.880-0.947$ & 0.940 & $0.905-0.966$ \\
\hline Puntuación total PAQ Damas & 0.899 & $0.850-0.935$ & 0.869 & $0.794-0.923$ \\
\hline Puntuación total PAQ Total & 0.913 & $0.887-0.937$ & 0.923 & $0.888-0.948$ \\
\hline
\end{tabular}

\section{DISCUSIONES}

Los resultados encontrados en esta investigación han sido favorables a las pretensiones de los investigadores, ya que este estudio ha mostrado una aceptable consistencia interna de los cuestionarios PAQ-C y PAQ-A, Ios cuales varían entre 0,84 y 0,85. Estos datos son comparables con los publicados por algunos autores, como Crocker (1997), valores de consistencia interna entre 0,79 y 0,89 aunque son superiores a los encontrados por Gómez et al. (2016) en escolares en altitud de 0,77, pero inferior al arrojados por García et al. (2019) de 0,97.

Muchas veces, la consistencia de respuesta de un cuestionario la termina por determinar el nivel de respuestas de los participantes, no la encuesta por sí sola. A pesar de que la encuesta presentada anteriormente tiene un valor por sobre lo que se considera aceptable, la posibilidad de ejecución de actividad física de quienes contestan la encuesta puede terminar generando diferencias individuales en las respuestas de los diferentes parámetros que esta posee, como los días de la semana. Además, las percepciones de los niños y adolescentes muchas veces pudieran ser exageradas en relación con tiempo y esfuerzo durante 
el desarrollo de actividades físicas y el número de días que suelen practicarla puede generar dificultades en la reconstrucción precisa del tiempo total que el niño o adolescente se mantiene activo (Miller, 2004). Además, se pueden generar instancias poco fiables al momento de responder a las encuestas, ya que un niño o adolescente puede pasar por procesos de imprecisión en el procesamiento cognitivo y recordatorio de las actividades que estaba realizando ayer, o hace cinco días atrás (Treuth, Hou, Young \& Maynard, 2005).

En cuanto a la fiabilidad test retest del coeficiente de correlación intraclase, fue ligeramente superior a los encontrados por Manchola-González, Bagur-Calafat, \& Girabent-Farrés (2017) (ICC=0.84) y por Martinez et al. (2009) (ICC=0.71) en su validación al castellano para el PAQ-A. Esto podría deberse a la rigurosidad en el protocolo utilizado en el que tanto el test como el retest, se realizaron exactamente en las mismas circunstancias, tanto de horario como de día de aplicación con una semana de diferencia.

En esta investigación, la fiabilidad test-retest se consideró aceptable, no existiendo grandes variaciones de porcentajes en ámbitos generales, por lo que este cuestionario puede ser respondido por niños que cursan desde un nivel escolar de 4to básico hasta adolescentes que cursan un nivel escolar de 4to medio, hablando del desarrollo cognitivo, esto refleja fielmente que poseen un desarrollo cognitivo óptimo para responder este tipo de cuestionarios.

\section{CONCLUSIONES}

Los cuestionarios PAQ-C y PAQ-A en su versión en español, tienen una consistencia interna más que aceptable, presentan una buena fiabilidad y reproductividad de test-retest, y contribuyen de forma segura y confiable a evaluar los niveles de actividad física de niños y adolescentes durante el periodo escolar anual de Chile. 


\section{REFERENCIAS BIBLIOGRÁFICAS}

Armstrong, N. \& Welsman, J. R. (2007). The Contribution of Physical Activity and Sedentary Behaviours to the Growth and Development of Children and Adolescents. Implications for Overweight and Obesity. Sports Medicine, 37(6), 1067-1086. DOI: http://doi.org/10.2165/00007256-200737060-00006

Caspersen, C; Powell, K; Chisterson, G. (1985). Physical activity, exercise and physical fitness: definitions and distinctions for health related research. Public Health Rep. 100(2): 126 -131.

Campo A. \& Oviedo H. (2008) Propiedades Psicométricas de una Escala: la Consistencia Interna. Rev. Salud pública. 10 (5), 831-839. Recuperado de https://www.redalyc.org/pdf/422/42210515.pdf

Crocker, P. R., Bailey, D. A., Faulkner, R. A., Kowalski, K. C., \& McGrath, R. (1997). Measuring general levels of physical activity: preliminary evidence for the Physical Activity Questionnaire for Older Children. Med Sci Sports Exerc. 29: 1344-1349. DOI: http://doi.org/10.1097/00005768-199710000-00011

Escalante, Y. (2011). Actividad física, ejercicio físico y condición física en el ámbito de la salud pública. Revista Española de Salud Pública, 85(4), 325-328. Recuperado de http://scielo.isciii.es/scielo.php?script=sci_arttext\&pid=S1135$57272011000400001 \&$ Ing=es\&tlng=es

Faúndez, C., Vásquez, J., Castillo, Marcelo, E., Souza, R., \& Castillo, F. (2019). Entrenamiento interválico aeróbico y de fuerza muscular en funcionarios universitarios obesos con ECNT: un estudio piloto. Nutrición clínica y Dietética Hospitalaria, 39(1):179-183. DOI: http://doi.org/10.12873/391faundez

Garcia, l., Muñoz, M., López, G., Gil, B., Puertas. M \& Gómez, A. (2019). Validación de un cuestionario sobre actitudes y práctica de Actividad Física y otros Hábitos Saludables mediante el método Delphi. Revista Española de Salud Pública, 93(16), 1-12. 
Faúndez, C. (2020). Fiabilidad y reproductividad de los Cuestionarios de Actividad Física PAQ-C Y PAQ-A en Estudiantes de Enseñanza Básica y Media de la Ciudad de Talca. UCMaule, 59, julio-diciem bre, 56-78.

DOI: http://doi.org/10.29035/ucmaule.59.56

Gobierno de Chile. (2017). Recomendaciones para la práctica de actividad física según curso de la Vida. Gobierno de Chile.

Gómez, R., Fuentes, J., Puño, L., de Arruda, M., \& Cossio, M. (2016). Reproductibilidad de un cuestionario que valora la actividad física en adolescentes escolares de altitud. Salud Uninorte. Barranquilla, 32(1), 95-104.

Janz, K. F., Dawson, J. D., \& Mahoney, L. T. (2000). Tracking physical fitness and physical activity from childhood to adolescence: the muscatine study. Medicine and Science in Sports and Exercise, 32(7), 1250-1257. DOI: http://doi.org/10.1097/00005768-200007000-00011

Kowalski, K., Crocker, P., \& Donen, R. (2004). The Physical Activity Questionnaire for Older Children (PAQ-C) and Adolescents (PAQ-A) Manual. Canada.

Lobstein, T., Baur, L., \& Uauy, R. (2004). Obesity in children and young people: a crisis in public health. Obesity reviews, 5(7), 4-85.

Manchola-González, J.; Bagur-Calafat, C. \& Girabent-Farrés, M. (2017). Fiabilidad de la versión española del cuestionario de actividad física PAQ-C. Revista Internacional de Medicina y Ciencias de la Actividad Física y el Deporte 17(65), 139-152. DOI: http://dx.doi.org/10.15366/rimcafd2017.65.008

Martínez, D., Martínez, V., Pozo, T., Villagra, A., Calle, M., Marcos, A. \& Veiga, O. (2009). Reliability and validity of the PAQ-A questionnaire to assess physical activity in spanish adolescents. Revista Española de Salud Pública, 83(3),427-39. DOI: http://doi.org/10.1590/s1135-57272009000300008

Miller, Y. (2004). Physical activity measurement in children by parental self-report. NSW Department of Health. Centre for Physical Activity and Health. Report No. CPAH 04-0001. NSW Center for Physical Activity and Health. Recuperado de https://www.health.nsw.gov.au/surveys/other/Documents/CPAHPhysical-activity-measurement-in-children-by-parental-self-report.pdf

National Center for Chronic Disease Prevention and Health Promotions, Centers for Disease Control and Prevention. (1997). Guidelines for school and 
Faúndez, C. (2020). Fiabilidad y reproductividad de los Cuestionarios de Actividad Física PAQ-C Y PAQ-A en Estudiantes de Enseñanza Básica y Media de la Ciudad de Talca. UCMaule, 59, julio-diciem bre, 56-78.

DOI: http://doi.org/10.29035/ucmaule.59.56

community programs to promote lifelong physical activity among young people. The Journal of School Health, 46(6), 1-36.

Organización Mundial de la Salud. (2010). Recomendaciones mundiales sobre actividad física para la salud. Organización Mundial de la Salud.

Parasuraman, A., Zeithaml, V. \& Berry, L. (1988). SERVQUAL: a multiple-item scale for measuring consumer perceptions of service quality. Journal of Retailing, 64(7). 12-37.

Perula de Torres, L. A., Lluch, C., Ruiz, R., Espejo, J., Tapia, G., \& Mengual, P. (1998). Prevalencia de actividad física y su relación con variables sociodemográficas y ciertos estilos de vida en escolares cordobeses. Revista Española de Salud Pública, 72(3), 233-244. Recuperado de http://scielo.isciii.es/scielo.php?script=sci_abstract\&pid=S113557271998000300008

Riddoch, C. J., Bo Andersen, L., Wedderkopp, N., Harro, M., Klasson-Heggebø, L., Sardinha, L. B.... Ekelund, U. (2004). Physical activity levels and patterns of 9- and 15-yr-old European children. Medicine and Sciences in Sports and Exercise, 36(1), 86-92. DOI: http://doi.org/10.1249/01.MSS.0000106174.43932.92

Sánchez, F., Campos, A., Vega de Carranza, M., Cortés, O., Esparza, M., Galbe, J., Gallego Iborra, A., García, J., Pallás, C., Rando, Á., San Miguel, M., Colomer, J. \& Mengual, J. (2019). Promoción de la actividad física en la infancia y la adolescencia (parte 1). Pediatría Atención Primaria, 27(83), 279-291. Recuperado de http://scielo.isciii.es/scielo.php?script=sci_arttext\&pid=S1139$76322019000300019 \&$ lng=es\&tlng=es

Serón, P., Muñoz, S. \& Lanas, F. (2010). Nivel de actividad física medida a través del cuestionario internacional de actividad física en población Chilena. Revista médica de Chile, 138(10), 1232-1239. DOI: https://dx.doi.org/10.4067/S0034-98872010001100004 
Faúndez, C. (2020). Fiabilidad y reproductividad de los Cuestionarios de Actividad Física PAQ-C Y PAQ-A en Estudiantes de Enseñanza Básica y Media de la Ciudad de Talca. UCMaule, 59, julio-diciem bre, 56-78.

DOI: http://doi.org/10.29035/ucmaule.59.56

Treuth, M., Hou, N., Young, D., Maynard, L. (2005). Validity and Reliability of the Fels Physical Activity Questionnaire for Children. Medicine and Science in Sports and Exercise. 37(3), 488-495. DOI: http://doi.org/10.1249/01.mss.0000155392.75790.83

Vidarte, J., Vélez, C., Sandoval, C. \& Alfonso, M. (2011). Actividad física: estrategia de promoción de salud. Hacia la Promoción de la Salud, Volumen 16(1), 202218.

Welk G. (2002). Physical activity assessment in health-related research. Human Kinetics. 


\section{ANEXO 1}

\section{CUESTIONARIO DE ACTIVIDAD FÍSICA PARA NIÑOS (PAQ-C)}

Nombre:

Sexo: $F$ M

Edad:

Curso:

Fecha de Nacimiento:

Queremos conocer cuál es tu nivel de actividad física en los últimos 7 días (última semana). Esto incluye todas aquellas actividades como deportes, gimnasia o danza que hacen sudar o sentirte cansado, o juegos que hagan que se acelere tu respiración como jugar al pilla-pilla, saltar la cuerda, correr, trepar y otras.

Recuerda:

1. No hay preguntas buenas o malas. Esto NO es un examen.

2. Contesta las preguntas de la forma más sincera posible. Esto es muy importante. 
Faúndez, C. (2020). Fiabilidad y reproductividad de los Cuestionarios de Actividad Física PAQ-C Y PAQ-A en Estudiantes de Enseñanza Básica y Media de la Ciudad de Talca. UCMaule, 59, julio-diciem bre, 56-78.

DOI: http://doi.org/10.29035/ucmaule.59.56

1. Actividad física en tu tiempo libre: ¿Has hecho alguna de estas actividades en los últimos 7 días (última semana)? Si tu respuesta es sí: ¿Cuántas veces las has hecho? (Marca un solo circulo por actividad).

\begin{tabular}{|l|l|l|l|l|l|}
\hline & NO & $\mathbf{1 - 2}$ & $\mathbf{3 - 4}$ & $\mathbf{5 - 6}$ & $\mathbf{7}$ VECES O MÁS \\
\hline Saltar la cuerda & & & & & \\
\hline Patinar & & & & & \\
\hline $\begin{array}{l}\text { Jugar a juegos como el pilla-pilla } \\
\text { (Pillada) }\end{array}$ & & & & & \\
\hline Montar en bicicleta & & & & & \\
\hline Caminar (como ejercicio) & & & & & \\
\hline Correr & & & & & \\
\hline Natación & & & & & \\
\hline Bailar danza & & & & & \\
\hline Bádminton & & & & & \\
\hline Rugby & & & & & \\
\hline Montar en monopatín & & & & & \\
\hline Fútbol/Futsal/Baby Futbol & & & & & \\
\hline Voleibol & & & & & \\
\hline Hockey & & & & & \\
\hline Basquetbol & & & & & \\
\hline Esquiar & & & & & \\
\hline Otros deportes de raqueta & & & & & \\
\hline Hándbol & & & & & \\
\hline Atletismo & & & & & \\
\hline Musculación/Pesas & & & & & \\
\hline Artes marciales (judo, karate, etc.) & & & & & \\
\hline Otros & & & & & \\
\hline
\end{tabular}


Faúndez, C. (2020). Fiabilidad y reproductividad de los Cuestionarios de Actividad Física PAQ-C Y PAQ-A en Estudiantes de Enseñanza Básica y Media de la Ciudad de Talca. UCMaule, 59, julio-diciem bre, 56-78.

DOI: http://doi.org/10.29035/ucmaule.59.56

2. En los últimos 7 días, durante las clases de educación física, ¿Cuántas veces estuviste muy activo durante las clases: jugando intensamente, corriendo, saltando, haciendo lanzamientos? (Señala sólo una).

\begin{tabular}{|l|l|}
\hline No hice/hago educación física & \\
\hline Casi nunca & \\
\hline Algunas veces & \\
\hline A menudo & \\
\hline Siempre & \\
\hline
\end{tabular}

3. En los últimos 7 días ¿Qué hiciste en el tiempo de recreo? (Señala sólo una).

\begin{tabular}{|l|l|}
\hline Estar sentado (hablar, leer, trabajo de clases) & \\
\hline Estar o pasear por los alrededores & \\
\hline Correr o jugar un poco & \\
\hline Correr y jugar bastante & \\
\hline Correr y jugar intensamente todo el tiempo & \\
\hline
\end{tabular}

4. En los últimos 7 días, ¿Qué hiciste hasta la comida (además de comer)? (Señala sólo una).

\begin{tabular}{|l|l|}
\hline Estar sentado (hablar, leer, trabajo de clases) & \\
\hline Estar o pasear por los alrededores & \\
\hline Correr o jugar un poco & \\
\hline Correr y jugar bastante & \\
\hline Correr y jugar intensamente todo el tiempo & \\
\hline
\end{tabular}

5. En los últimos 7 días, ¿Cuántos días después del colegio hiciste deporte, baile o jugaste a juegos en los que estuvieras muy activo? (Señala sólo una).

\begin{tabular}{|l|l|}
\hline Ninguno & \\
\hline 1 vez en la última semana & \\
\hline $2-3$ veces en la última semana & \\
\hline 4 veces en la última semana & \\
\hline 5 veces o más en la última semana & \\
\hline
\end{tabular}


Faúndez, C. (2020). Fiabilidad y reproductividad de los Cuestionarios de Actividad Física PAQ-C Y PAQ-A en Estudiantes de Enseñanza Básica y Media de la Ciudad de Talca. UCMaule, 59, julio-diciem bre, 56-78.

DOI: http://doi.org/10.29035/ucmaule.59.56

6. En los últimos 7 días, ¿Cuántas tardes hiciste deporte, bailar o jugar a juegos en los que estuviste muy activo? (Señala sólo una).

\begin{tabular}{|l|l|}
\hline Ninguno & \\
\hline 1 vez en la última semana & \\
\hline 2-3 veces en la última semana & \\
\hline 4-5 veces en la última semana & \\
\hline 6-7 veces en la última semana & \\
\hline
\end{tabular}

7. El último fin de semana. ¿Cuántas veces hiciste deporte, baile o jugar a juegos en los que estuvieras muy activo? (Señala sólo una).

\begin{tabular}{|l|l|}
\hline Ninguno & \\
\hline 1 vez & \\
\hline $2-3$ veces & \\
\hline $4-5$ veces & \\
\hline 6 o más veces & \\
\hline
\end{tabular}

8. ¿Cuál de las siguientes frases describen mejor tu última semana? Lee las cinco antes de decidir cuál te describe mejor. (Señala sólo una).

Todo o la mayoría de mi tiempo libre lo dediqué a actividades que suponen poco esfuerzo físico

Algunas veces (1 02 veces la última semana) hice actividades físicas en mi tiempo libre (por ejemplo, hacer deportes, correr, nadar, montar en bicicleta, hacer aeróbic)

A menudo (3-4 veces la última semana) hice actividad físicas en mi tiempo libre

Bastante a menudo (5-6 veces la última semana) hice actividad físicas en mi tiempo libre

Muy a menudo (7 o más veces la última semana) hice actividad físicas en mi tiempo libre 
Faúndez, C. (2020). Fiabilidad y reproductividad de los Cuestionarios de Actividad Física PAQ-C Y PAQ-A en Estudiantes de Enseñanza Básica y Media de la Ciudad de Talca. UCMaule, 59, julio-diciem bre, 56-78.

DOI: http://doi.org/10.29035/ucmaule.59.56

9. Señala con qué frecuencia hiciste actividad física para cada día de la última semana (como hacer deportes, jugar, bailar, o cualquier otra actividad física).

\begin{tabular}{|l|l|l|l|l|l|}
\hline & Ninguna & Poca & Normal & Bastante & Mucha \\
\hline Lunes & & & & & \\
\hline Martes & & & & & \\
\hline Miércoles & & & & & \\
\hline Jueves & & & & & \\
\hline Viernes & & & & & \\
\hline Sábado & & & & & \\
\hline Domingo & & & & & \\
\hline
\end{tabular}

10. ¿Estuviste enfermo esta última semana o algo impidió que hicieras normalmente actividades físicas? (Señala sólo una).

\begin{tabular}{|l|l|}
\hline $\mathrm{Si}$ & \\
\hline No & \\
\hline
\end{tabular}

Si la respuesta es sí, ¿Qué impidió? 
Faúndez, C. (2020). Fiabilidad y reproductividad de los Cuestionarios de Actividad Física PAQ-C Y PAQ-A en Estudiantes de Enseñanza Básica y Media de la Ciudad de Talca. UCMaule, 59, julio-diciem bre, 56-78.

DOI: http://doi.org/10.29035/ucmaule.59.56

\section{CUESTIONARIO DE ACTIVIDAD FÍSICA PARA ADOLESCENTES (PAQ-A)}

Nombre:

Edad:

Sexo: F M

Curso:

Fecha de Nacimiento:

Queremos conocer cuál es tu nivel de actividad física en los últimos 7 días (última semana). Esto incluye todas aquellas actividades como deportes, gimnasia o danza que hacen sudar o sentirte cansado, o juegos que hagan que se acelere tu respiración como jugar al pilla-pilla, saltar a la comba, correr, trepar y otras.

Recuerda:

1. No hay preguntas buenas o malas. Esto NO es un examen.

2. Contesta las preguntas de la forma más honesta y sincera posible. Esto es muy importante. 
Faúndez, C. (2020). Fiabilidad y reproductividad de los Cuestionarios de Actividad Física PAQ-C Y PAQ-A en Estudiantes de Enseñanza Básica y Media de la Ciudad de Talca. UCMaule, 59, julio-diciem bre, 56-78.

DOI: http://doi.org/10.29035/ucmaule.59.56

1. Actividad Física en tu tiempo libre: ¿Has hecho alguna de estas actividades en los últimos 7 días (última semana)? Si tu respuesta es sí, ¿cuántas veces las has hecho? (Marca un solo círculo por actividad).

\begin{tabular}{|l|l|l|l|l|l|}
\hline & NO & $\mathbf{1 - 2}$ & $\mathbf{3 - 4}$ & $\mathbf{5 - 6}$ & $\mathbf{7}$ VECES O MÁS \\
\hline Saltar a la cuerda & & & & & \\
\hline Patinar & & & & & \\
\hline $\begin{array}{l}\text { Jugar a juegos como el pilla-pilla o } \\
\text { pillada }\end{array}$ & & & & & \\
\hline Montar en bicicleta & & & & & \\
\hline Caminar (como ejercicio) & & & & & \\
\hline Correr & & & & & \\
\hline Aeróbic/spinning & & & & & \\
\hline Bailar/danza & & & & & \\
\hline Bádminton & & & & & \\
\hline Rugby & & & & & \\
\hline Montar en monopatín & & & & & \\
\hline Fútbol / Futsal / Baby Futbol & & & & & \\
\hline Voleibol & & & & & \\
\hline Hockey & & & & & \\
\hline Basquetbol & & & & & \\
\hline Esquiar & & & & & \\
\hline Otros deportes de raqueta & & & & & \\
\hline Hándbol & & & & & \\
\hline Atletismo & & & & & \\
\hline Musculación / pesas & & & & & \\
\hline Artes marciales (judo, kárate, etc.) & & & & & \\
\hline Otros & & & & & \\
\hline
\end{tabular}

2. En los últimos 7 días, durante las clases de educación física, ¿cuántas veces estuviste muy activo durante las clases: jugando intensamente, corriendo, saltando, haciendo lanzamientos? (Señala sólo una).

\begin{tabular}{|l|l|}
\hline No hice / hago educación física & \\
\hline Casi nunca & \\
\hline Algunas veces & \\
\hline A menudo & \\
\hline Siempre & \\
\hline
\end{tabular}


Faúndez, C. (2020). Fiabilidad y reproductividad de los Cuestionarios de Actividad Física PAQ-C Y PAQ-A en Estudiantes de Enseñanza Básica y Media de la Ciudad de Talca. UCMaule, 59, julio-diciem bre, 56-78.

DOI: http://doi.org/10.29035/ucmaule.59.56

3. En los últimos 7 días ¿qué hiciste normalmente a la hora de la comida (antes y después de comer)? (Señala sólo una).

\begin{tabular}{|l|l|}
\hline Estar sentado (hablar, leer, trabajo de clase) & \\
\hline Estar o pasear por los alrededores & \\
\hline Correr o jugar un poco & \\
\hline Correr y jugar bastante & \\
\hline Correr y jugar intensamente todo el tiempo & \\
\hline
\end{tabular}

4. En los últimos 7 días, inmediatamente después de la escuela hasta las 6, ¿cuántos días jugaste a algún juego, hiciste deporte o bailes en los que estuvieras muy activo? (Señala sólo una).

\begin{tabular}{|l|l|}
\hline Ninguno & \\
\hline 1 vez en la última semana & \\
\hline $2-3$ veces en la última semana & \\
\hline 4 veces en la última semana & \\
\hline 5 veces o más en la última semana & \\
\hline
\end{tabular}

5. En los últimos 7 días, ¿cuántos días a partir de media tarde (entre las 6 y las 10) hiciste deportes, baile o jugaste a juegos en los que estuvieras muy activo? (Señala sólo una).

\begin{tabular}{|l|l|}
\hline Ninguno & \\
\hline 1 vez en la última semana & \\
\hline $2-3$ veces en la última semana & \\
\hline 4 veces en la última semana & \\
\hline 5 veces o más en la última semana & \\
\hline
\end{tabular}

6. El último fin de semana, ¿cuántas veces hiciste deportes, baile o jugar a juegos en los que estuviste muy activo? (Señala sólo una).

\begin{tabular}{|l|l|}
\hline Ninguno & \\
\hline 1 vez en la última semana & \\
\hline $2-3$ veces en la última semana & \\
\hline 4 veces en la última semana & \\
\hline 5 veces o más en la última semana & \\
\hline
\end{tabular}


Faúndez, C. (2020). Fiabilidad y reproductividad de los Cuestionarios de Actividad Física PAQ-C Y PAQ-A en Estudiantes de Enseñanza Básica y Media de la Ciudad de Talca. UCMaule, 59, julio-diciem bre, 56-78.

DOI: http://doi.org/10.29035/ucmaule.59.56

7. ¿Cuál de las siguientes frases describen mejor tu última semana? Lee las cinco antes de decidir cuál te describe mejor. (Señala sólo una).

Todo o la mayoría de mi tiempo libre lo dediqué a actividades que suponen poco esfuerzo físico

Algunas veces (1 o 2 veces) hice actividades físicas en mi tiempo libre (por ejemplo, hacer deportes, correr, nadar, montar en bicicleta, hacer aeróbic)

A menudo (3-4 veces a la semana) hice actividad física en mi tiempo libre

Bastante a menudo (5-6 veces en la última semana) hice actividad física en mi tiempo libre

Muy a menudo (7 o más veces en la última semana) hice actividad física en mi tiempo libre

8. Señala con qué frecuencia hiciste actividad física para cada día de la semana (como hacer deporte, jugar, bailar o cualquier otra actividad física).

\begin{tabular}{|l|l|l|l|l|l|}
\hline & Ninguna & Poca & Normal & Bastante & Mucha \\
\hline Lunes & & & & & \\
\hline Martes & & & & & \\
\hline Miércoles & & & & & \\
\hline Jueves & & & & & \\
\hline Viernes & & & & & \\
\hline Sábado & & & & & \\
\hline Domingo & & & & & \\
\hline
\end{tabular}

9. ¿Estuviste enfermo esta última semana o algo impidió que hicieras normalmente actividades físicas?

\begin{tabular}{|l|l|}
\hline Sí & \\
\hline No & \\
\hline
\end{tabular}

Si la respuesta es sí, ¿Qué impidió? 